\title{
Synthesis of different-sized gold nanostars for Raman bioimaging and photothermal therapy in cancer nanotheranostics
}

\author{
Jie Gao ${ }^{1,2}$, Maria Sanchez-Purra ${ }^{3}$, Hao Huang ${ }^{4}$, Shunhao Wang ${ }^{1,2}$, Yunan Chen ${ }^{1,2}$, Xuefeng Yu, \\ Qian Luo ${ }^{4}$, Kimberly Hamad-Schifferli ${ }^{3,5^{*}}$ \& Sijin Liu ${ }^{1,2 *}$ \\ ${ }^{1}$ State Key Laboratory of Environmental Chemistry and Ecotoxicology, Research Center for Eco-Environmental Sciences, \\ Chinese Academy of Sciences, Beijing 100085, China \\ ${ }^{2}$ University of Chinese Academy of Sciences, Beijing 100049, China \\ ${ }^{3}$ Department of Engineering, University of Massachusetts Boston, Boston, MA 02125, United States \\ ${ }^{4}$ Institute of Biomedicine and Biotechnology, Shenzhen Institutes of Advanced Technology, Chinese Academy of Sciences, \\ Shenzhen 518055, China \\ ${ }^{5}$ Department of Mechanical Engineering, Massachusetts Institute of Technology, Cambridge, MA 02139, United States
}

Received April 10, 2017; accepted May 23, 2017; published online July 28, 2017

\begin{abstract}
Gold nanoparticles (AuNPs) have been attractive for nanomedicine because of their pronounced optical properties. Here, we customerized the methods to synthesize two types of gold nanostars, Au nanostars-1 and Au nanostars-2, which have different spire lengths and optical properties, and also spherical AuNPs. Compared to nanospheres, gold nanostars were less toxic to a variety of cells, including macrophages. Au nanostars-1 and Au nanostars-2 also manifested a similar pattern of tissue distribution upon in vivo administration in mice to that of nanospheres, and but reveled less liver retention than nanospheres. Due to their strong absorption in the near-infrared (NIR), Au nanostars-2 induced a strong hyperthermia effect in vitro upon excitation at $808 \mathrm{~nm}$, and elicited a robust photothermal therapy (PTT) efficacy in ablating tumors in a mouse model of orthotopic breast cancer using 4T1 breast cancer cells. Meanwhile, Au nanostars-1 showed a great capability to enhance the Raman signal through surface-enhanced Raman spectroscopy (SERS) in 4T1 cells. Our combined results opened a new avenue to develop Au nanostars for cancer imaging and therapy.
\end{abstract}

gold nanoparticle, nanostar, surface plasmon resonance, tissue distribution, toxicity profile

Citation: Gao J, Sanchez-Purra M, Huang H, Wang S, Chen Y, Yu X, Luo Q, Hamad-Schifferli K, Liu S. Synthesis of different-sized gold nanostars for Raman bioimaging and photothermal therapy in cancer nanotheranostics. Sci China Chem, 2017, 60: 1219-1229, doi: 10.1007/s11426-017-9088-x

\section{Introduction}

Because of their facile synthesis, feasible biocompatibility, chemical stability and versatility in chemical modification, $\mathrm{Au}$ nanoparticles (AuNPs) have been promising in a myriad of fields. AuNPs of different shapes (e.g. nanorods, nanocages and nanostars) have a size- and shape-tunable surface plasmon resonance (SPR) band, resulting in a strong

*Corresponding authors (email: Kim.Hamad@umb.edu; sjliu@rcees.ac.cn) optical absorption throughout the visible and near-infrared (NIR) spectral region [1-3]. Furthermore, AuNPs offer a strong local electromagnetic field to enhance the Raman signals of nearby molecules, resulting in surface-enhanced Raman scattering (SERS) [4-6]. Consequently, AuNPs have gained considerable attention for a wide spectrum of biomedical applications, including bio-imaging, drug/gene delivery, bio-sensing and photothermal therapy (PTT) [2,6-11].

Thus far, sphere-like and rod-like AuNPs have been comprehensively investigated and characterized for various biomedical applications [12-14]. In contrast, star-shaped 
AuNPs, which are star-shaped branched nanocrystals $[13,15-17]$, are relatively new in comparison to their spherical counterparts. Their morphology gives them unique optical and physicochemical properties, which can be manipulated even with small shape modifications. Moreover, the sharp tips confer Au nanostars with a tunable, narrow SPR band and also strong electromagnetic enhancement effects. A single type of nanomaterial that can be imaged by multiple orthogonal approaches, also with diagnostic capabilities and effective therapy are highly desirable, but remain challenging. The optical properties of Au nanostars can be exploited for both PTT and Raman bioimaging, and thus have the potential to act as multifunctional theranostics. To this end, $\mathrm{Au}$ nanostars are emerging as a promising nanotheranostics platform for diagnostic and therapeutic purposes [18-20].

Because of their tremendous potential for nanomedicine, their toxicity and biodistribution need to be explored [21,22]. Prior studies of Au nanospheres and nanorods have found that the toxicological properties of AuNPs are influenced by their physical and bio-interface properties, where particle shape, size, and surface chemistry can impact their toxicity, biodistribution [23,24], and pharmacokinetics [25]. Hence, investigation of Au nanostars both in vitro and in vivo toxicities need to be determined in order to best exploit them for biomedical applications.

Here, we studied $\mathrm{Au}$ nanostars with two different spire lengths and also spherical AuNPs. In order to have better understanding of the biological ramifications of AuNPs, the toxicity effects of $\mathrm{Au}$ nanostars should be studied both in vitro and in vivo. First, we compared the toxicity effects of $\mathrm{Au}$ nanostars with $\mathrm{Au}$ nanospheres. We examined the biodistribution of the particles in vivo in a mouse model. Furthermore, we demonstrated their ability to be used for Raman imaging of cancer cells using conjugated Raman reporters, and also in vivo NIR PTT.

\section{Materials and method}

\subsection{Materials}

$\mathrm{Au}$ chloride trihydrate was purchased from Sigma-Aldrich (CAS: 16961-25-4) (USA). Bis(sulphatophenyl)phenylphosphine dehydrate (BPS), was purchased from Aldrich (CAS: 308103-66-4) (USA). N-(2-hydroxyethyl) piperazine- $N$ '-(2-ethanesulphonic acid) (HEPES) was purchased from United States Biochemical Company (CAT: 16926). Sodium citrate dehydrate was purchased from Mallinckrodt Chemicals (CAS: 6132-04-3, USA). Au nanostars with different extinction spectra were synthesized by tuning the $\mathrm{Au} / \mathrm{HEPES}$ ratio in solution. We mixed 10 and $22.5 \mathrm{~mL}$ of $140 \mathrm{mM}$ HEPES with 15 and $2.5 \mathrm{~mL}$ of $18 \mathrm{M} \Omega$ deionized (Milli-Q) water, for $\mathrm{Au}$ nanostars-1 and for $\mathrm{Au}$ nanostars- 2 , respectively, followed by the addition of $400 \mu \mathrm{L}$ of
$10 \mathrm{mg} / \mathrm{mL} \mathrm{HAuCl}_{4} \cdot 3 \mathrm{H}_{2} \mathrm{O}$ and further vortexing. After vortexing, solutions sat undisturbed for $1 \mathrm{~h}$, during which the $\mathrm{Au}$ nanostars crystallized. Afterwards, $\sim 0.5 \mathrm{mg}$ BPS was added for nanostar stabilization, and the solution was vortexed and left undisturbed for $1 \mathrm{~h}$. After this time, the nanostars were ready to be used in experiments. The nanostars were separated from excess reagents by centrifugation at $12000 \mathrm{r} / \mathrm{min}$ for $12 \mathrm{~min}$. The resulting nanostar pellet was re-suspended in $1 \mathrm{~mL}$ of $18 \mathrm{M} \Omega$ water.

\subsection{Synthesis of AuNPs}

For the synthesis of Au nanospheres, $49.5 \mathrm{~mL}$ of water (MilliQ) were mixed with $500 \mu \mathrm{L}$ of $10 \mathrm{mg} / \mathrm{mL} \mathrm{HAuCl}_{4} \cdot 3 \mathrm{H}_{2} \mathrm{O}$ and brought to a boil. Then, $0.5 \mathrm{~mL}$ of sodium citrate dihydrate $(10 \mathrm{mg} / \mathrm{mL})$ and temperature was kept for $15 \mathrm{~min}$. Afterwards, the heat was turned off and the sample was stirred for $15 \mathrm{~min}$ more, when it was the transferred to a cold stirrer. After $30 \mathrm{~min}, 0.5 \mathrm{mg}$ of BPS were added to the mixture and sample was further stirred overnight.

\subsection{Characterization of AuNPs}

The AuNPs were prepared for transmission electron microscope (TEM) imaging by dropping down sample solutions onto carbon-coated copper electron microscopy grids (Beijing Zhongjingkeyi Technology Co., Ltd., China) and allowed to dry at $25^{\circ} \mathrm{C}$ overnight. TEM analysis was performed on an H-7500 transmission electron microscope (Hitachi Scientific Instruments, Japan). Meanwhile, the UV-Vis spectra were recorded on a Cary $100 \mathrm{UV}-\mathrm{Vis}$ spectrophotometer (Agilent Technologies, USA) in $1 \mathrm{~cm}$ quartz cuvettes over the range from 200 to $800 \mathrm{~nm}$. A Zetasizer Nano ZS from Malvern Instruments (UK) was used to measure the hydrodynamic diameter $\left(D_{\mathrm{H}}\right)$ by dynamic light scattering (DLS) and the $\zeta$ of the AuNPs.

\subsection{Cell culture}

All cell lines were obtained from the Shanghai Cell Bank of Type Culture Collection of China. Murine macrophage cell line J774A.1, mouse breast cancer cell line 4T1, human prostate cancer cell line PC3, human hepatic carcinoma cell line HepG2, mouse liver cancer cell line Hepa1-6 were routinely cultured in recommended medium (Hyclone/Gibco) supplemented with $10 \%$ fetal bovine serum (FBS; Gibco) and $100 \mathrm{U} / \mathrm{mL}$ penicillin streptomycin (Hyclone) within wet atmosphere with $5 \% \mathrm{CO}_{2}$ at $37^{\circ} \mathrm{C}$, as previously described [26].

\subsection{Cytotoxicity/cell viability assay}

Cytotoxicity/cell viability was determined using the MTT (3-(4,5-dimethylthiazol-2-yl)-2,5-diphenyltetrazolium bromide) assay. Briefly, $1 \times 10^{4}$ cells/well were seeded in 96-well 
plates, and were thereafter incubated in a humidified 5\% $\mathrm{CO}_{2}$-balanced air incubator at $37^{\circ} \mathrm{C}$. After $12 \mathrm{~h}$, cells were treated with AuNPs at 1,10 and $20 \mu \mathrm{g} / \mathrm{mL}$ after $24 \mathrm{~h}$. The cytotoxicity/cell viability was assayed with the MTT reagent following the instructions provided by the manufacturers (Shanghai Sangon Biological Engineering Technology and Service Co., China). Absorbance values were monitored at $595 \mathrm{~nm}$ on a microplate reader.

\subsection{ATP production assay}

Intracellular adenosine triphosphate (ATP) concentration was determined using an ATP assay kit following the manufacturer's instructions (Promega, USA). In brief, $1 \times 10^{4}$ cells/well were seeded in 96-well plates, and were cultured overnight, followed by AuNPs treatment for $24 \mathrm{~h}$. Finally, the CellTiter-Glo solution $(100 \mu \mathrm{L})$ was added to each well, followed by incubation for $10 \mathrm{~min}$, and the absorbance at $450 \mathrm{~nm}$ was recorded on a Varioskan Flash Multimode plate reader (Thermo Scientic, USA).

\subsection{LDH release assay}

Leakage of lactate dehydrogenase (LDH) from cells was assayed with a LDH assay kit according to manufacturer's instructions (Nanjing Jiancheng, China). In short, $1 \times 10^{4}$ cells/well were seeded in 96-well plate, and were then treated with AuNPs at 1,10 and $20 \mu \mathrm{g} / \mathrm{mL}$ for $24 \mathrm{~h}$. Afterwards, cell culture medium was collected after treatment and was then centrifuged at $12000 \mathrm{r} / \mathrm{min}$. The supernatants were used for the assay of LDH release according to the protocol provided by the manufacturer. Absorbance was measured at $490 \mathrm{~nm}$ on a plate reader.

\subsection{Cellular localization of AuNPs through TEM}

For TEM analysis, $2 \times 10^{6}$ J774A.1 cells were first inoculated in $6 \mathrm{~cm}$ plates. Then, cells were exposed to AuNPs at $10 \mu \mathrm{g} / \mathrm{mL}$ for $24 \mathrm{~h}$, followed by cell collection after phosphate buffer saline (PBS) washing. The harvested cells were fixed with $2.5 \%$ glutaraldehyde solution and embedded with epoxy resin. The ultrathin cell specimens $(70 \mathrm{~nm})$ were thereafter prepared on the grids, stained with $1 \%$ lead citrate and $0.5 \%$ urinal acetate and finally examined using a high-resolution JEOL JEM 2010F transmission electron microscope (Hitachi Scientific Instruments, Japan).

\subsection{Animal experimentation}

Female BALB/c mice (7-8 weeks old with body weight around $20 \mathrm{~g}$ ) were purchased from the Vital River laboratories (Beijing, China), and were thereafter housed in a specific pathogen-free and aseptic animal center. All animals were maintained and used in accordance with the Guidelines of the Animal Ethics Committee at the Research Center for Eco-Environmental Sciences, Chinese Academy of Sciences.
For tissue distribution and biocompatibility assays, mice were administrated with AuNPs through a single intra-tail vein injection in $200 \mu \mathrm{L}$ at indicated concentrations $(n=3$ mice per group). For the control group, mice received PBS only. Mice were sacrificed $24 \mathrm{~h}$ after AuNPs administration.

\subsection{Quantitation of AuNPs through ICP-MS}

Prior to cellular treatment, $2 \times 10^{6} \mathrm{~J} 774 \mathrm{~A} .1$ cells were seeded in $6 \mathrm{~cm}$ plates. Then, cells were exposed to AuNPs at $10 \mu \mathrm{g} / \mathrm{mL}$ for $24 \mathrm{~h}$, and cells were collected post treatment. Thereafter, collected cells were digested with mixed acid using a microwave on a MARS machine (CEM Corp., Mattews, NC). For tissue distribution, various tissues were collected after mice were sacrificed. Tissues were similarly digested. Finally, digested samples were thereafter subjected to inductively coupled plasma mass spectrometry (ICP-MS) on an Agilent 7500 instrument (Agilent, Tokyo, Japan).

\subsection{Histological examination}

Tissue specimens were collected when mice were sacrificed, and fixed in 10\% formalin diluted in PBS. Fixed tissues were embedded in paraffin blocks, and then cut into $5 \mu \mathrm{m}$ thick sections onto slides. Hematoxylin and eosin (H\&E) staining was carried out following the standard protocol. Slides were examined with an Axio Scope A1 optical microscope (Carl Zeiss, Inc., Germany).

\subsection{CBC analysis of peripheral blood}

First, $20 \mu \mathrm{L}$ fresh blood was collected from each mouse, and diluted into $2 \mathrm{~mL}$ standard dilution buffer provided by the manufacturer. Thereafter, the diluted blood samples were assessed on a hematology analyzer (Nihon Kohden, MEK7222K, Japan).

\subsection{Photothermal assessment in vitro}

Aqueous suspensions of AuNPs $(100 \mu \mathrm{L}$, at various particle concentrations) were loaded into a 96-well plate, and were then irradiated with a diode laser $(\lambda=808 \mathrm{~nm})$ from the top at a density of $1 \mathrm{~W} / \mathrm{cm}^{2}$. A NIR camera (ICI7320, Infrared Camera Inc., Beaumont, TX, USA) was placed on top of the plate, and thermographs were continuously recorded by the NIR camera at an interval of $5 \mathrm{~s}$. The obtained data of thermographs were processed and analyzed with the IR Flash software (Version 2.10, Infrared Camera Inc.) to gain the average temperature of each sample at different time points.

\subsection{In vivo PTT examination of AuNPs in mice}

First, the mouse model of orthotopic breast cancer was established by injecting 5000 4T1 cells into the fourth mammary fat pads (MFPs) after a midline incision was made, as described in our recent studies $[27,28]$. When tumors were 
grown to a size of $\sim 100 \mathrm{~mm}^{3}$, the PTT experiment was carried out. In short, mice bearing a similar size of tumors were randomly divided into four groups: (1) PBS; (2) PBS+laser (the sham group); (3) Au nanostars-2 only; (4) Au nanostars-2+laser Animals were anesthetized with $2 \%$ isoflurane (ISoFlo, Abott Laboratories) in $100 \%$ oxygen and placed in a prone position on a table. Mice were administrated through intra-tumor injection of Au nanostars-2 $(30 \mu \mathrm{g})$ or PBS only. Right after injection, the entire region covering the tumor was irradiated to a diode laser $(808 \mathrm{~nm})$ at a power density of $0.6 \mathrm{~W} / \mathrm{cm}^{2}$. During irradiation, thermographs were taken using a NIR camera, as described above. Tumor growth was monitored for another $15 \mathrm{~d}$. The tumor size was determined using the formula, as volume $=$ length $\times(\text { width })^{2} / 2$.

\subsection{Preparation of Raman probes}

The Raman probes were prepared by adding 4-MBA $\left(10 \mu \mathrm{L}, 10^{-3} \mathrm{M}\right)$ to $3 \mathrm{~mL}$ AuNPs solutions at $80 \mu \mathrm{g} / \mathrm{mL}$, and the mixtures were incubated overnight, followed by centrifugation at $8000 \mathrm{r} / \mathrm{min}$ for $10 \mathrm{~min}$. The excess 4-MBA molecules in the supernatants were removed, and the prepared probes were finally dispersed in $3 \mathrm{~mL}$ DI water.

\subsection{Raman mapping}

The Au nanoastars- 1 probes were exposed to $4 \mathrm{~T} 1$ cells for $6 \mathrm{~h}$. Then, the cells were fixed on cover glass by using $4 \%$ paraformaldehyde. Raman spectra and Raman mapping were acquired on a Horiba Jobin-Yvon Lab Ram HR-VIS high-resolution confocal Raman microscope equipped with a $633 \mathrm{~nm}$ laser as the excitation source at room temperature.

\subsection{Statistical analysis}

Experimental data were shown in mean \pm standard deviation (SD). Statistical analysis was performed using the Student's $t$-test. $P$ values less than $0.05(P<0.05)$ were determined to be statistically significant in the current study.

\section{Results and discussion}

\subsection{Synthesis and characterization of AuNPs}

We investigated the effect of shape on toxicity and biocompatibility, hence spherical nanoparticles (nanospheres) and nanostars were examined. In addition, we wanted to probe the effect of curvature, therefore we examined two different nanostars that differed in arm length. Two shapes of $\mathrm{Au}$ nanostars, here termed Au nanostars-1 and Au nanostars-2, were synthesized using $\mathrm{HAuCl}_{4}$ reduction with HEPES [29].

As shown in Figure 1(a), the transmission electron microscopy (TEM) images revealed star-like multi-branched morphology of $\mathrm{Au}$ nanostars-1 and $\mathrm{Au}$ nanostars-2, with increased distance from the middle of the longest axis to the intersection of two longest axes of the star for Au nanostars-2 relative to Au nanostars-1, in analogy to our previous results [29]. DLS was used to measure their mean hydrodynamic diameter $\left(D_{\mathrm{H}}\right)$, which was 37 and $35 \mathrm{~nm}$ for Au nanostars-1 and nanostars-2, respectively. Au nanostars' physicochemical properties, functionality and biocompatibility were compared to Au nanospheres. Au nanospheres had a mean core diameter of $\sim 30 \mathrm{~nm}$ (Figure 1(a)), as reflected by the TEM analysis, and a mean $D_{\mathrm{H}}$ of $27 \mathrm{~nm}$, determined by DHL analysis (Figure 1(b)). All three AuNPs samples were homogeneously dispersed, and showed favorable polydispersity and stability in water and cell culture medium without significant agglomeration for $24 \mathrm{~h}$. The zeta-potential analysis showed that all AuNPs were similarly negatively charged (Figure 1(c)), consistent with our past studies [1]. Furthermore, the UV-Vis-NIR spectra showed absorption peaks for $\mathrm{Au}$ nanostars-1, Au nanostars- 2 and nanospheres at $\sim 620$, 740 and $525 \mathrm{~nm}$, respectively (Figure 1(d)).

\subsection{Cytotoxicity assessment of AuNPs}

We first assessed the cytotoxicity of the Au nanostars and Au nanospheres by multiple assays. Macrophages are the most important sentinel cells in the body responding to invading microorganisms and particulates including nanomaterials by carrying out phagocytosis and releasing pro-inflammatory cytokines [30,31]. We chose J774A.1 macrophages for cellular treatment with AuNPs. Cell viability was not significantly affected for J774A.1 cells upon Au nanostars-1, Au nanostars- 2 , and nanospheres at 1,10 and $20 \mu \mathrm{g} / \mathrm{mL}$ for $24 \mathrm{~h}$, relative to the untreated control, as reflected by the MTT assay $(P>0.05$, Figure 2(a)). Levels of LDH release were also unchanged upon exposure to the AuNPs, further confirming the negligible toxicity to J774A.1 cells (Figure 2(b)). Afterwards, a more sensitive cytotoxicity assay, ATP synthesis [32], was employed to determine possible effects on cellular metabolism in response to these AuNPs. The cellular ATP levels were not significantly changed in cells after exposure to the AuNPs at 1 and $10 \mu \mathrm{g} / \mathrm{mL}$ compared to untreated cells; however, a significant reduction of ATP production $(\sim 20 \%$ decline) was observed at $20 \mu \mathrm{g} / \mathrm{mL}$ (Figure 2(c), $P<0.05$ ). Importantly, a further $10 \%$ drop of ATP production was found in cells treated with nanospheres at $20 \mu \mathrm{g} / \mathrm{mL}$ (Figure 2(c)), compared to cells treated with Au nanostars- 1 and Au nanostars-2 (Figure 2(c), $P<0.05$ ). These data implied that nanostars had a slightly less effect on cell viability than nanospheres in J774A. 1 macrophages.

We explored the effect of the AuNPs on additional cell types, including 4T1, HepG-2, Hepa1-6, and PC-3 cells. All of the AuNPs did not significantly impair the cell viability of 4T1 cells compared to the untreated control (Figure 3(a)), as characterized by the MTT assay $(P>0.05)$ with only a slight 
(a)

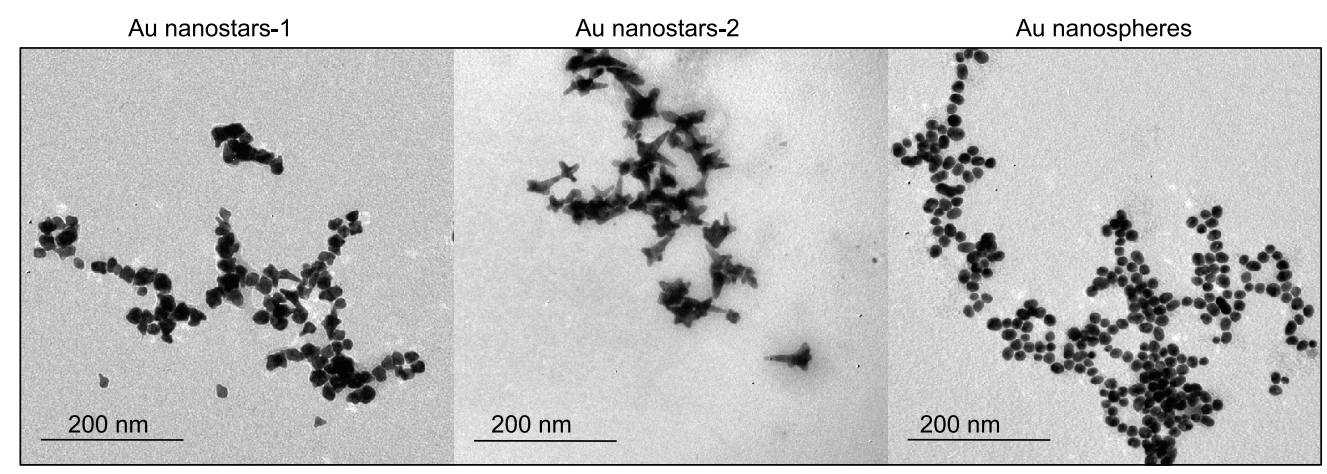

(b)

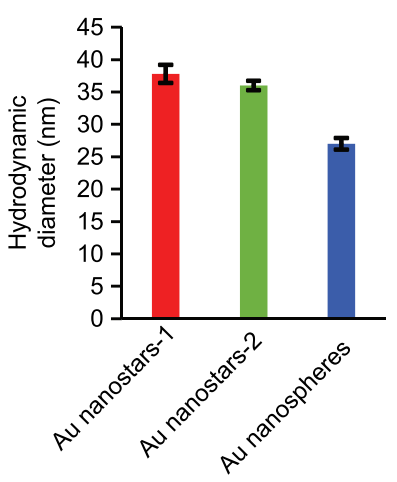

(c)

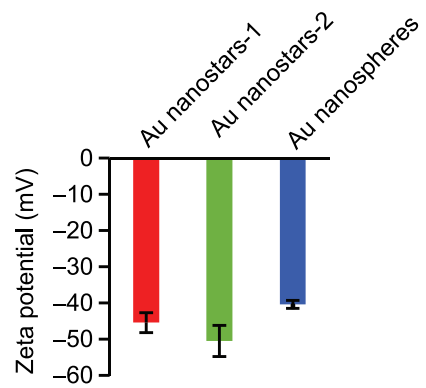

(d)
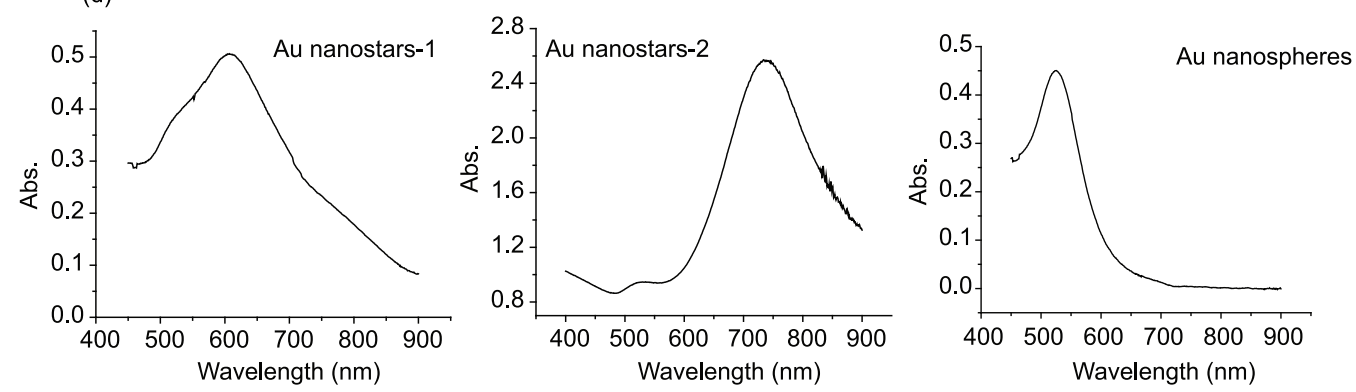

Figure 1 Physical characterization of AuNPs. (a) TEM images of Au nanostars-1, Au nanostars-2 and Au nanospheres; (b) hydrodynamic diameters of AuNPs measured by DLS; (c) zeta-potentials of AuNPs solutions; (d) UV-Vis spectra of Au nanostars-1, Au nanostars-2 and Au nanospheres in water (color online).

reduction at $20 \mu \mathrm{g} / \mathrm{mL}$. Furthermore, the ATP production measurements revealed a dose-dependent decline of cellular ATP production in 4T1 cells with the increase of AuNPs concentration from 1 to $20 \mu \mathrm{g} / \mathrm{mL}(P<0.05)$. At $20 \mu \mathrm{g} / \mathrm{mL}$, ATP levels were reduced by $\sim 50 \%$ (Figure 3 (b), $P<0.001$ ). Of note, nanospheres induced a greater repression on ATP production than nanostars, especially at $20 \mu \mathrm{g} / \mathrm{mL}$ (Figure 3(b)). Since no significant difference was found between $\mathrm{Au}$ nanostars-1 and Au nanostars- 2 in suppressing cellular ATP synthesis, $\mathrm{Au}$ nanostars-1 and nanospheres were further compared in HepG2, Hepa1-6 and PC-3 cells. In agreement with the above results, nanospheres incurred slightly greater inhibition of ATP production compared to Au nanostars-1, as evidenced by further decline of cellular ATP levels in HepG2, Hepa1-6 and PC-3 cells responded to nanospheres at 10 and $20 \mu \mathrm{g} / \mathrm{mL}$, compared to those in Au nanostars-1-treated cells (Figure 3(c), $P<0.05$ ). Collectively, our combined data suggested that all AuNPs induced minimal cytotoxicity to a variety of cells, where the Au nanostars had a less impact on cell viability.

\subsection{Cellular uptake and intracellular localization of AuNPs}

AuNPs have a strong optical absorption due to their surface plasmon resonance (SPR) [33], which builds up the basis for biological applications. Their ability to efficiently convert light to heat conversion efficacy, could be altered by internalization of particles by cells in biological settings [25,34]. To this end, we also probed the cellular uptake of AuNPs by 

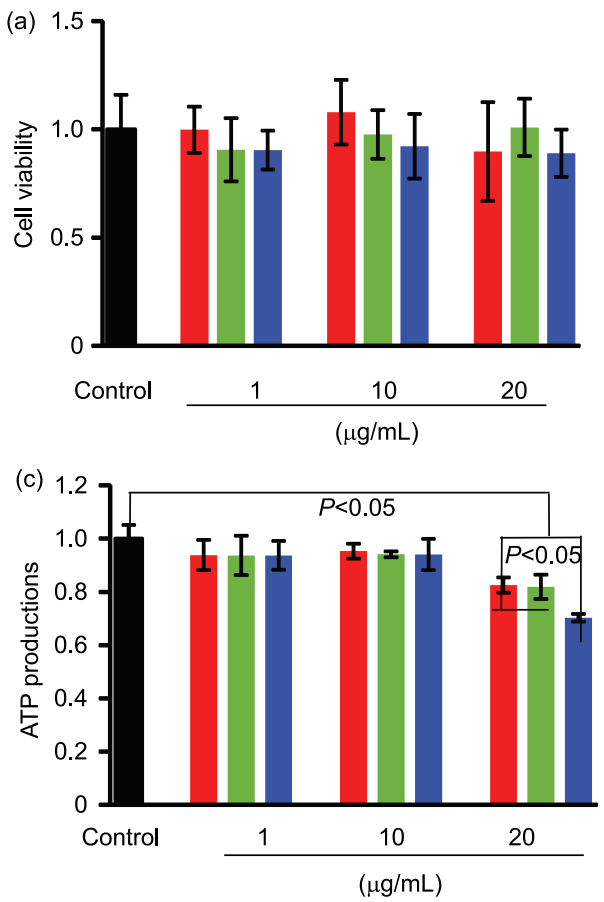

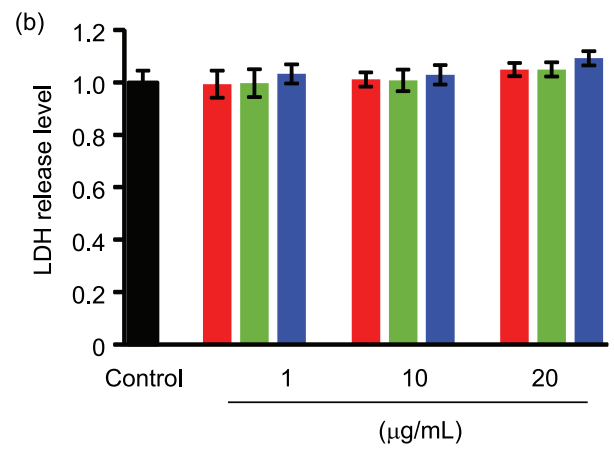

Contro

Au Nanostars-1

Au Nanostars-2

Au Nanospheres

Figure 2 Cytotoxicity evaluation upon AuNPs in J774A.1 cells. (a) Cell viability determined by the MTT assay in J774A.1 cells upon exposure to AuNPs at 1,10 and $20 \mu \mathrm{g} / \mathrm{mL}$ for $24 \mathrm{~h}(n=6)$; (b) relative LDH release in J774A.1 cells treated with AuNPs for $24 \mathrm{~h}(n=6)$; (c) cellular energy metabolism in J774A.1 cells, as characterized by ATP production, in response to AuNPs for $24 \mathrm{~h}$. Cellular ATP mass was assayed by the bioluminescent intensity on a microplate reader and relative ATP levels were quantified by normalizing to the untreated control $(n=6)$ (color online).
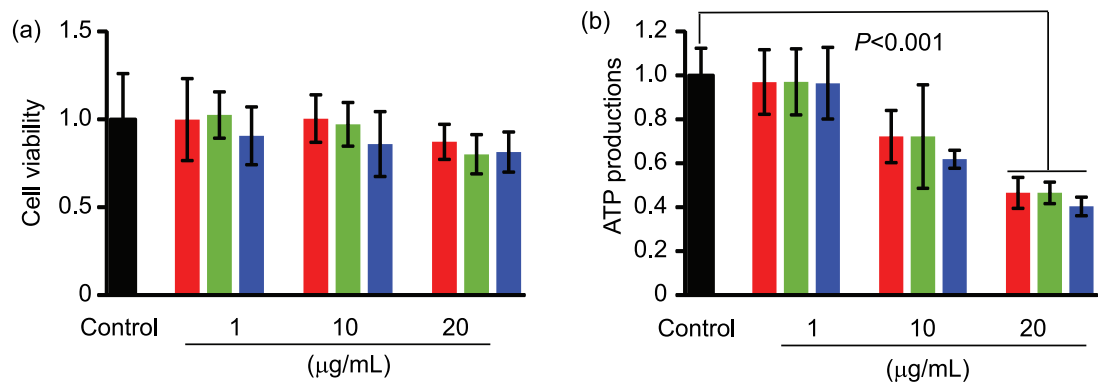

Control

Au Nanostars-1

Au Nanostars-2

Au Nanospheres

(c)

\begin{tabular}{|c|c|c|c|c|c|c|c|}
\hline \multirow{2}{*}{ Assay } & \multirow{2}{*}{ Cell type } & \multicolumn{3}{|c|}{ Au nanostars -1} & \multicolumn{3}{|c|}{ Au nanospheres } \\
\hline & & $1 \mu \mathrm{g} / \mathrm{mL}$ & $10 \mu \mathrm{g} / \mathrm{mL}$ & $20 \mu \mathrm{g} / \mathrm{mL}$ & $1 \mu \mathrm{g} / \mathrm{mL}$ & $10 \mu \mathrm{g} / \mathrm{mL}$ & $20 \mu \mathrm{g} / \mathrm{mL}$ \\
\hline \multirow{3}{*}{ ATP } & HepG-2 & - & $\downarrow$ & $\downarrow \downarrow$ & - & $\downarrow$ & $\downarrow \downarrow \downarrow$ \\
\hline & Hepa1-6 & - & - & $\downarrow$ & - & $\downarrow$ & $\downarrow$ \\
\hline & $P G 3$ & - & - & $\downarrow \downarrow$ & - & $\downarrow \downarrow$ & $\downarrow \downarrow \downarrow$ \\
\hline
\end{tabular}

Figure 3 Cytotoxicity determination in 4T1 cells responded to AuNPs. (a) Cytotoxicity determined by the MTT assay in 4T1 cells treated with AuNPs at concentrations of 1, 10 and $20 \mu \mathrm{g} / \mathrm{mL}$ for $24 \mathrm{~h}(n=6)$; (b) ATP production in 4T1 cells upon AuNPs for $24 \mathrm{~h}(n=6)$; (c) ATP production levels quantified after cells were treated with AuNPs at various concentrations for $24 \mathrm{~h}(n=6)$. Arrows represent the drop, compared to untreated control: one arrow, $20 \%$ reduction; two arrows, $\sim 40 \%$ reduction; three arrows, $\sim 60 \%$ reduction (color online).

ICP-MS. Macrophages, as the most cardinal phagocytes, are capable of ingesting invading particles [30,31]. We thus quantified the uptake of AuNPs in J774A.1 macrophages by measuring the Au content in the cell lysates post treatment. Using ICP-MS (Figure 4(a)), the total intracellular $\mathrm{Au}$ mass was comparable among cells treated with $\mathrm{Au}$ nanostars-1, Au nanostars-2 and nanospheres, with approx- imately $4 \mu \mathrm{g}$ per $1 \times 10^{4}$ cells after exposure to $10 \mu \mathrm{g} / \mathrm{mL}$ for $24 \mathrm{~h}$. Nonetheless there was a slight increase of the $\mathrm{Au}$ mass in nanospheres-treated cells in comparison to nanostars-treated cells, possibly due to enhanced cellular uptake of sphere-like particles by cells relative to particles in other shapes $[35,36]$. Furthermore, TEM imaging substantiated the intracellular localization of AuNPs. As shown in Figure 4(b), 
(a)

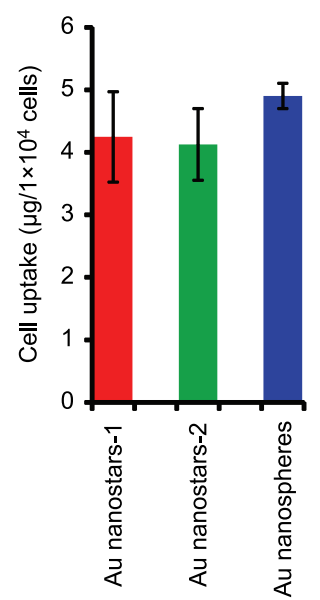

(b)

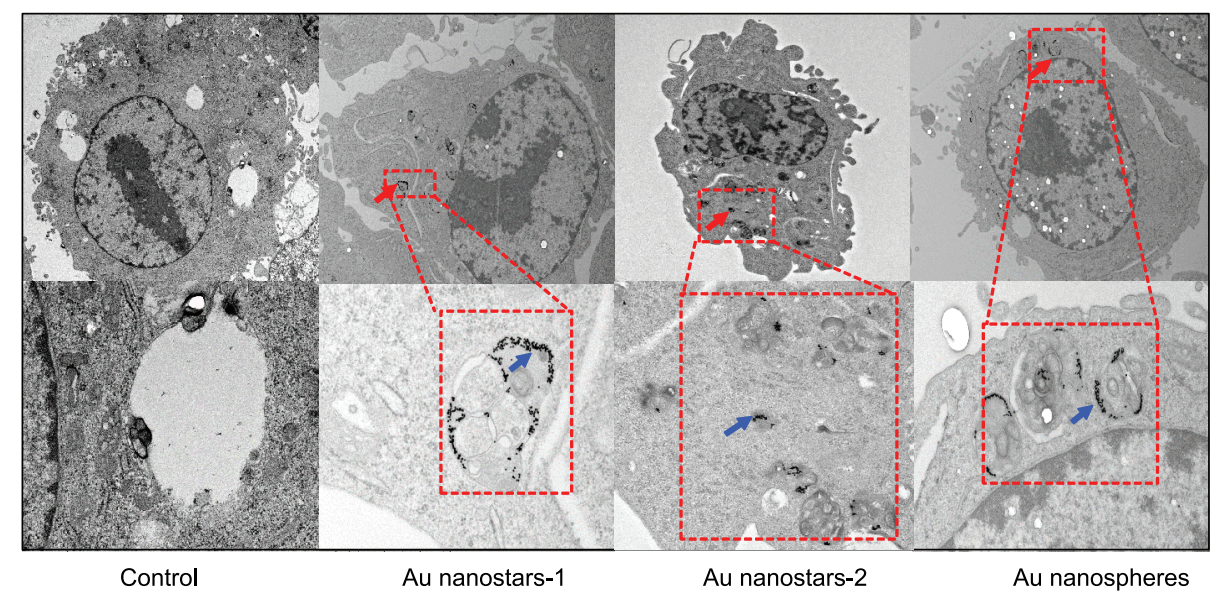

Figure 4 Cellular uptake and the localization of AuNPs. (a) Quantification of the amount of elemental Au in cells by ICP-MS in J774.1A cells after 24-h incubation with $10 \mu \mathrm{g} / \mathrm{mL}$ AuNPs $(n=6)$; (b) TEM images of J774.1A cells post treatment with AuNPs. After incubation with $10 \mu \mathrm{g} / \mathrm{mL}$ Au nanostars-1 and Au nanospheres for $24 \mathrm{~h}$, cells were collected for TEM analysis of intracellular AuNPs. Blue arrows denote nanoparticles, and red arrows indicate phagosomes (color online).

AuNPs were readily visualized in the cytosol (mostly within membrane bound structures, i.e. phagosomes) in J774A.1 cells upon Au nanostars-1 and Au nanospheres, consistent with previous studies [25].

\subsection{In vivo distribution and biocompatibility assessment of AuNPs in mice}

Due to their superfine size, nanomaterials can travel through the blood stream to target [37], be taken up by cells [8], and interface directly with biomolecules, such as proteins [38] and DNA [39]. In order to achieve a safe and sustainable use of nanoparticles, biodistribution measurements are necessary to evaluate their kinetic metabolism, selective targeting efficacy and toxicity profiles [40]. Therefore, we investigated the accumulation of AuNPs in various organs from mice after intravenous administration of $\mathrm{Au}$ nanostars-1, Au nanostars-2 and $\mathrm{Au}$ nanospheres through elemental Au quantification by ICP-MS of the digested tissues. The liver and spleen were the predominant sites for AuNPs accumulation, in agreement with previous studies (Figure 5(a)) [41,42]. However, Au nanostars- 2 and Au nanostars- 1 showed reduced liver retention by $\sim 25 \%$ and $50 \%$ compared to that of nanospheres, in parallel to the compromised cellular uptake of nanostars by macrophages. This finding implied a longer circulation time for the $\mathrm{Au}$ nanostars, highlighting a reduced likelihood for them to cause hepatoxicity.

Regarding the in vivo safety profiles, complete blood count (CBC) analysis of peripheral blood from mice following administration of these AuNPs did not identify significant alterations to various lineages of blood cells, including red blood cells, platelets and myeloid cells (data not shown). Moreover, histological examination of lung, liver, heart, kidney and spleen manifested no significant histological alterations for these tissues through H\&E staining of tissue sections (Figure 5(b)). These results together recognized a preferential biocompatibility of these AuNPs in vivo.
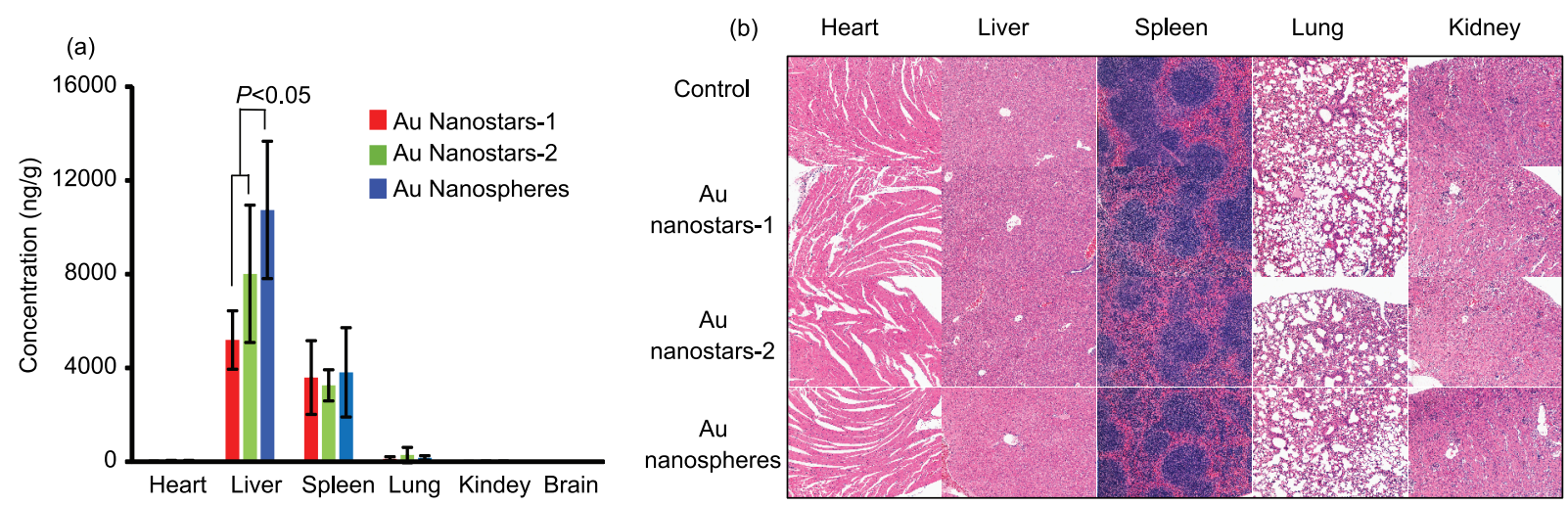

Figure 5 Biodistrbution and histological examinations of tissues upon AuNPs in vivo. (a) Biodistribution of AuNPs in various organs in mice after AuNPs administration at $10 \mu \mathrm{g} /$ mouse for $24 \mathrm{~h}$. (b) H\&E staining of various tissues from mice administrated with AuNPs at $10 \mu \mathrm{g} / \mathrm{mice}$ for $24 \mathrm{~h}$. Original magnification, $\times 100$ (color online). 


\subsection{Photothermal efficacy in vitro}

Plasmonic materials with a strong NIR absorption are considered to be good photothermal agents for cancer therapy [3]. Thus, we investigated the photothermal properties of $\mathrm{Au}$ nanostars-2 due to their strong absorption in the NIR. First, we measured the temperature increase of an aqueous dispersion of Au nanostars-2 under $808 \mathrm{~nm}$ NIR cw laser irradiation, which is a wavelength that coincides with the tissue window and also the nanostar LSPR peak. The temperature of the $\mathrm{Au}$ nanostars- 2 solution increased by $26^{\circ} \mathrm{C}$ after 10 min of irradiation, indicating that the Au nanostars- 2 can rapidly and efficiently convert NIR light into thermal energy (Figure 6(a)). Furthermore, by means of a reported method, the photothermal conversion efficiency $(\eta)$ of the Au nanostars-2 was determined to be approximately $\sim 13.0 \%[43,44]$. The good photothermal ability of Au nanostars- 2 with low toxicity indicated that they had the outstanding potential to act as photothermal agents.

To investigate the phototoxicity effects of the Au nanostars- 2 for biomedical applications, the toxicity of Au nanostars- 2 was measured at different concentrations $(0,10,20$ and $40 \mu \mathrm{g} / \mathrm{mL}$ ) by treating $4 \mathrm{~T} 1$ cells for $24 \mathrm{~h}$ and probing the cell viability using the MTT assay. Cell viability decreased rapidly by $\sim 30 \%$ at concentrations above $20 \mu \mathrm{g} / \mathrm{mL}$ under laser irradiation of $808 \mathrm{~nm} 1 \mathrm{~W} / \mathrm{cm}^{2}$ for $10 \mathrm{~min}$ at the LSPR (red, Figure 6(b)). An even greater reduction of cell viability $(>90 \%)$ was observed in cells treated with Au nanostars- 2 at concentrations above $20 \mu \mathrm{g} / \mathrm{mL}$ with laser irradiation (Figure 6(b), $P<0.001$ ). At the same time, the decrease of cell viability without the laser excitation was much less than with it (Figure 6(b)). These data demonstrated that the NIR irradiation of the Au nanostars- 2 could efficiently kill of cancer cells.

\subsection{Photothermal efficacy in vivo}

Since Au nanostars-2 elicited a marked hyperthermia effect

(a)

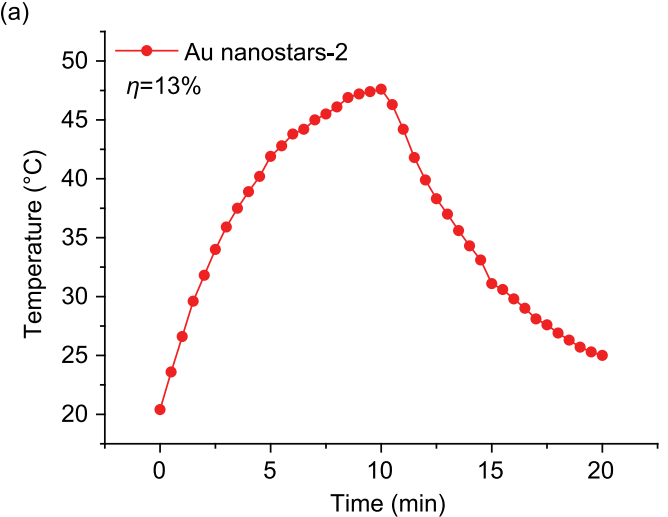

in vitro, we next evaluated the ability of Au nanostars- 2 to ablate breast tumors in vivo. We first established a mouse model of orthotopic breast cancer using 4T1 cells as described in our previous reports $[27,28]$. When the tumor size reached $100 \mathrm{~mm}^{3}$, we applied PTT with intra-tumor injection of Au nanostars-2 (808 nm, $0.6 \mathrm{~W} / \mathrm{cm}^{2}$ for $\left.5 \mathrm{~min}\right)$. During the irradiation, we adjusted the spot laser beam to cover the whole tumor region. Real-time temperature tracking of mice using an infrared thermal camera was an effective tool to monitor the treatment process. Thermal imaging (Figure 7(a)) showed that an irradiation-induced temperature increase was observed at the orthotopic breast tumors over the time course post administration of Au nanostars- 2 . The maximum temperature inside the tumors that could be reached was found to be as high as $55^{\circ} \mathrm{C}$, sufficient to kill tumor cells [45-48]. The tumor size and body weights were checked every day for $15 \mathrm{~d}$ (Figure 7(b, c)). Tumor growth in mice was substantially repressed with NIR laser irradiation relative to the sham group $(P<0.001$, Figure $7(\mathrm{~d}))$. The final tumor size reached approximately $1800 \mathrm{~mm}^{3}$ in untreated mice (red, Figure 7(b)), whereas the final tumors were nearly not tangible in Au nanostars-2/NIR-treated mice (area in red dashed circles, Figure 7(d)). Nonetheless, the PTT treatment did not cause a decrease in the body weight of treated mice, relative to untreated mice (Figure $7(\mathrm{c}), P>0.05$ ). The results indicated that the Au nanostars- 2 could be effectively applied for PTT in breast orthotopic tumors. This study would also open a new avenue in the development of therapeutics for cancer based on the NIR effect of Au nanostars.

\subsection{Raman bioimaging in $4 \mathrm{~T} 1$ breast cancer cells}

Probing living cells with Raman spectroscopic techniques has great value for studying the molecular and cellular response of nanomaterials used in theranostic applications [20,49,50]. In SERS, a metallic nanostructure reacts with incident photons, resulting in an enhanced electric field in the vicinity of

(b)

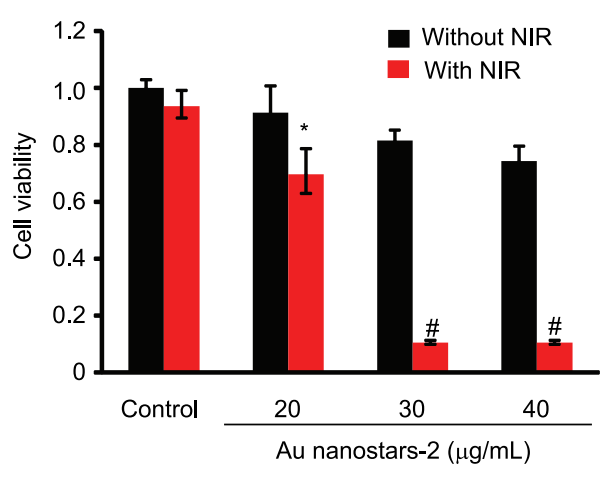

Figure 6 The photothermal effect of Au nanostars-1 in vitro. (a) The photothermal effect curve of the temperature of an Au nanostars- 1 solution in water under NIR irradiation for $10 \mathrm{~min}$; (b) cytotoxicity of 4T1 cells at the different concentrations of Au nanostars-1 with or without NIR determined by the MTT assay $(n=6)$ (color online). 

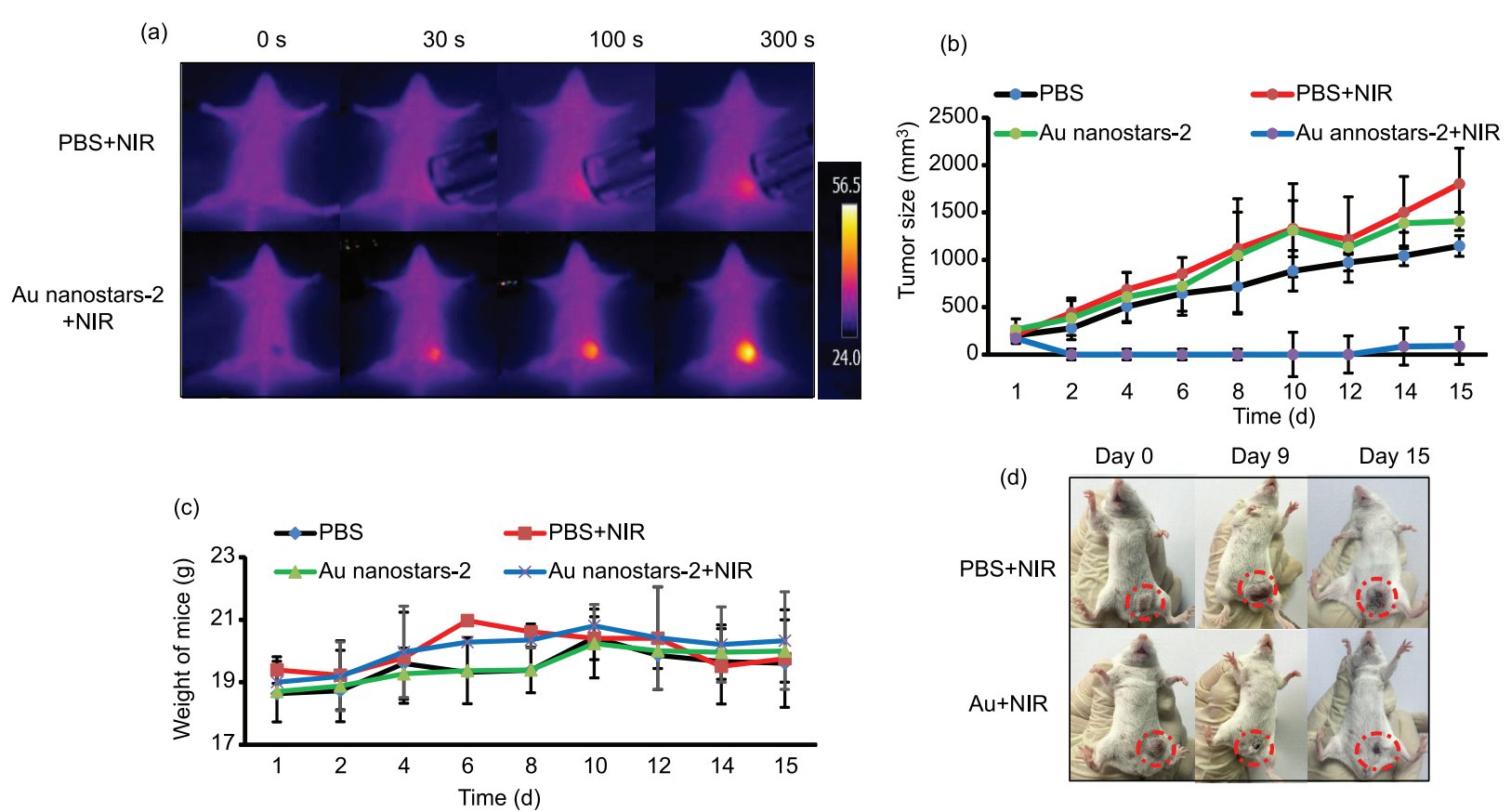

Figure 7 Hyperthermia effect and photothermal ablation of tumors in mice. (a) Laser irradiation temperature elevation in 4T1 tumors implanted in mice after injection of nanostars-1. The temperature was recorded at different time intervals after material injection. (b) The tumor growth curves in mice upon PTT with nanostars- 1 over the time course $(n=3)$. (c) The alterations of body weight of mice upon PTT with nanostars- 1 over time $(n=3)$. (d) The representative images of tumors post PTT at different time times post injection (color online).

the metal surface. Once a target molecule is within a few nanometers of the surface of a metallic nanostructure, Raman scattering can be enhanced by orders of magnitude [19,51]. SERS has been extensively investigated in numerous biological applications due to the high-sensitivity detection limit and selective molecule reactions. Consistent with previous findings [52,53], Au nanostars-1 display two SPR bands in visible and NIR region due to the particle spherical core and its branches, respectively. According to their optical spectra (Figure 1), Au nanospheres and Au nanostars-1 have absorption in the near-infrared region, so we investigated the ability of using the Au nanostars for SERS imaging. 4-Mercaptobenzoic acid (4-MBA), a widely-used Raman reporter [54], was conjugated to the particles to result in a SERS probe where the 4-MBA signal could be used to map the particles. To conjugate the 4-MBA to the particles, $10 \mu \mathrm{L}$ of a 4-MBA $10^{-3} \mathrm{M}$ solution was respectively added to $3 \mathrm{~mL} \mathrm{Au}$ nanospheres and $\mathrm{Au}$ nanostars-1 solution at $80 \mu \mathrm{g} / \mathrm{mL}$ and incubated overnight, allowing the thiol on the reporter to bind to the particles. Then, we assessed the SERS spectra of as-prepared $\mathrm{Au}$ nanospheres and $\mathrm{Au}$ nanostars- 1 probes by dropping $10 \mu \mathrm{L}$ of the probe solution onto a Si substrate. The resulting Raman spectra with a $633 \mathrm{~nm}$ laser excitation showed peaks consistent with previous reports [54] (Figure 8(a)). The two characteristic vibrational modes at 1074 and $1585 \mathrm{~cm}^{-1}$ can be attributed to the ring-breathing modes of the molecule. Compared with Au nanospheres, Au nanostars-1 exhibited enhanced Raman signals of conjugated 4-MBA molecules, which can be attributed to the lightning rod effect from the spires in $\mathrm{Au}$ nanostars-1. Using these prominent SERS features, we then investigated the capability of Au nanostars-1 as probes for the imaging of 4T1 cancer cells post Au nanostars-1 treatment (Figure 8(b)). Representative Raman spectra acquired from inside the cell at the different locations outside and inside of the cell, indicative of point 1-4 (the phase-contrast image) were obtained (Figure 8(c)). It was observed that each signal was non-interfering, which was accounted for by the narrow spectral features of the 4-MBA Raman peaks. To obtain a Raman map of a single cell, we used a $1 \mathrm{~mW}$ laser power density and $1 \mathrm{~s}$ integration time per point, and the characteristic peak of $1585 \mathrm{~cm}^{-1}$ was chosen to form the SERS mapping. The bright field image and corresponding overlaid Raman image of a single 4T1 breast cell treated with probes are shown in Figure 8(b). The enhanced signal (in red and yellow color) indicated the distribution of probes inside the cell, and confirmed that the tags after endocytosis were located almost exclusively in the cytoplasm. Collectively, our data suggested that Au nanostars-1-MBA can be an attractive nanomaterial for cancer imaging by Raman and phototherapy.

\section{Conclusions}

In the current study, we investigated the diverse facets of $\mathrm{Au}$ nanostars in cancer nanotheranostics. Au nanostars-2 harbored a remarkable capability to induce hyperthermia which 
(a)

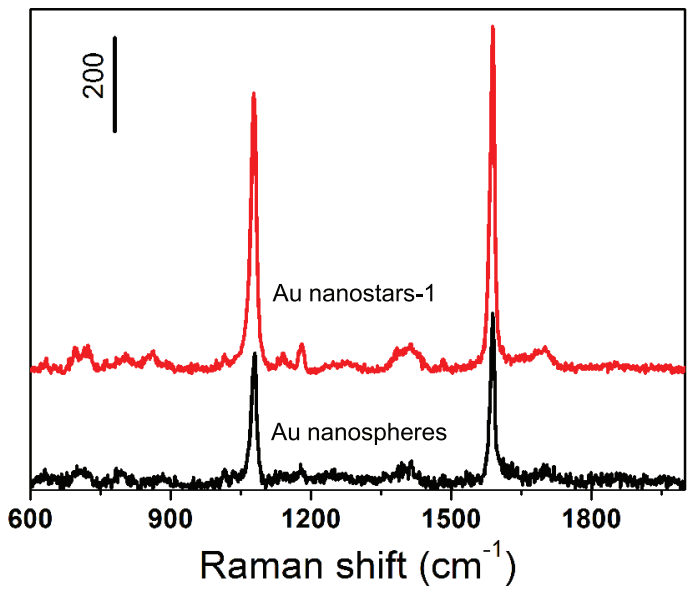

(c)

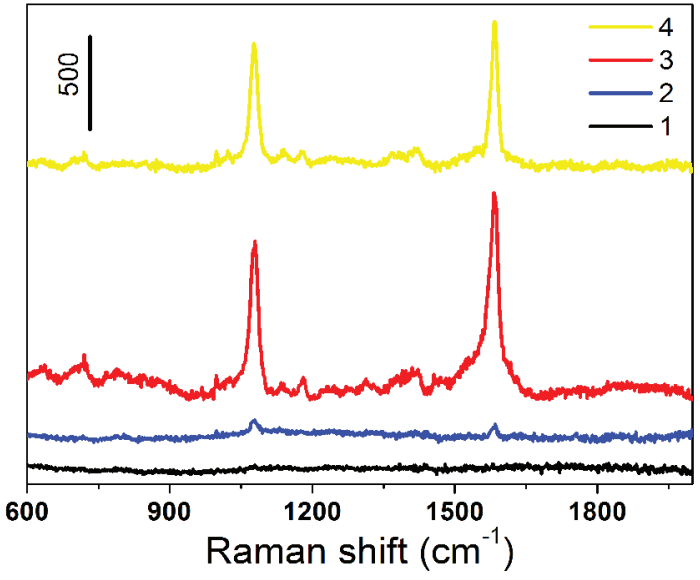

(b)
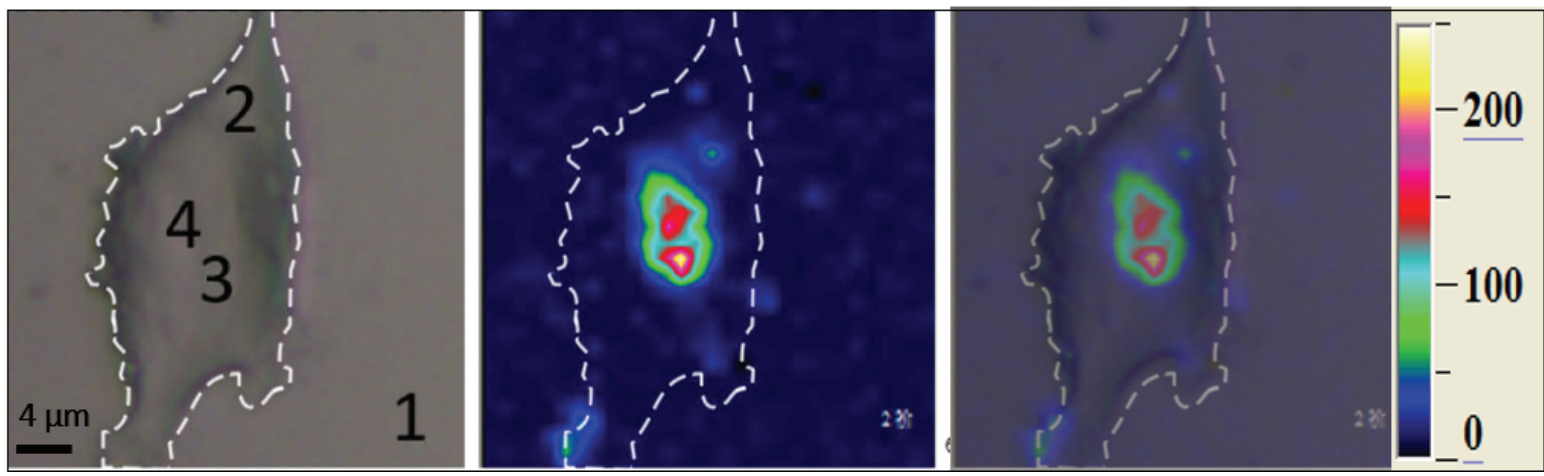

Figure 8 Raman spectra of nanostars-1 in 4T1 cells. (a) Raman spectra of Au nanostars-1 and Au nanospheres incubated with 4-MBA overnight; (b, c) a representative Raman bright-field image of a 4T1 cell after exposure to with nanostars-1-MBA for $24 \mathrm{~h}$ was selected for streamline mapping and the corresponding Raman spectra. Points 1-4 indicated different locations inside or outside of the selected cell (color online).

could be utilized to ablate tumors. Meanwhile, Au nanostars- 1 could be applied for use in SERS imaging due to their ability to enhance Raman signals in breast cancer cells. Au nanostars were also demonstrated to be less toxic than Au nanospheres in a few cell types. We also measured the in vivo biodistribution of $\mathrm{Au}$ nanostars, and found that $\mathrm{Au}$ nanostars showed less liver retention mass compared to $\mathrm{Au}$ nanospheres. Thus, our study demonstrated that Au nanostars are promising in cancer thermotherapy and imaging with improved biocompatibility and reduced liver retention. Both of these findings can aid in the design of nanomaterials for therapeutic or diagnostic applications for improved cytoand biocompatibility, which has been a significant barrier for clinical use of nanomaterials. Gold nanoparticles of varying shapes have been attractive for their optical properties, which can be leveraged for through-tissue imaging such as SERS but also photoacoustic imaging, as well as light-induced therapies, such as photothermal and photodynamic therapy and also triggered drug release. Therefore these results can aid in improving these important theranostic applications.

Acknowledgments This work was supported by the National Basic Research Program (2014CB932000), the Strategic Priority Research Program of the Chinese Academy of Sciences (XDB14000000) and the National Natural Science Foundation of China $(21425731,21637004)$.

Conflict of interest The authors declare that they have no conflict of interest.

1 Kim JE, Choi JH, Colas M, Kim DH, Lee H. Biosens Bioelectron, 2016, 80: 543-559

2 Yang X, Yang M, Pang B, Vara M, Xia Y. Chem Rev, 2015, 115: 10410-10488

3 Huang X, Jain PK, El-Sayed IH, El-Sayed MA. Lasers Med Sci, 2008, 23: $217-228$

4 Zhou H, Qiu C, Yu F, Yang H, Chen M, Hu L, Sun L. J Phys Chem C, 2011, 115: 11348-11354

5 Tąta A, Szkudlarek A, Kim Y, Proniewicz E. Spectrochim Acta A-Mol Biomol Spectrosc, 2017, 173: 251-256

6 Liu XL, Liang S, Nan F, Yang ZJ, Yu XF, Zhou L, Hao ZH, Wang QQ. Nanoscale, 2013, 5: 5368-5374

7 Palonpon AF, Ando J, Yamakoshi H, Dodo K, Sodeoka M, Kawata S, Fujita K. Nat Protoc, 2013, 8: 677-692

8 Weissleder R, Nahrendorf M, Pittet MJ. Nat Mater, 2014, 13: 125-138

9 Ju HX. Sci China Chem, 2011, 54: 1202-1217

10 Wang P, Wan Y, Ali A, Deng S, Su Y, Fan C, Yang S. Sci China Chem, 2016, 59: 237-242

11 Feng B, Zhou F, Wang D, Xu Z, Yu H, Li Y. Sci China Chem, 2016, 
59: 984-990

12 Alkilany AM, Nagaria PK, Hexel CR, Shaw TJ, Murphy CJ, Wyatt MD. Small, 2009, 5: 701-708

13 Kumar PS, Pastoriza-Santos I, Rodriguez-Gonzalez B, Garcia de Abajo FJ, Liz-Marzan LM. Nanotechnology, 2007, 19: 015606

14 Zhou CH, Gan LL, Zhang YY, Zhang FF, Wang GZ, Jin L, Geng RX. Sci China Ser B-Chem, 2009, 52: 415-458

15 Song HM, Wei Q, Ong QK, Wei A. ACS Nano, 2010, 4: 5163-5173

16 Fales AM, Yuan H, Vo-Dinh T. Langmuir, 2011, 27: 12186-12190

17 Yu J, Guo WC, Yang M, Luan Y, Tao JZ, Zhang XW. Sci China Chem, 2014, 57: 1211-1217

18 Rodríguez-Lorenzo L, Krpetic Z, Barbosa S, Alvarez-Puebla RA, Liz-Marzán LM, Prior IA, Brust M. Integr Biol, 2011, 3: 922-926

19 Jimenez de Aberasturi D, Serrano-Montes AB, Langer J, Henriksen-Lacey M, Parak WJ, Liz-Marzán LM. Chem Mater, 2016, 28: 6779-6790

20 Vendrell M, Maiti KK, Dhaliwal K, Chang YT. Trends Biotech, 2013, 31: 249-257

21 Wilhelm S, Tavares AJ, Dai Q, Ohta S, Audet J, Dvorak HF, Chan WCW. Nat Rev Mater, 2016, 1: 16014

22 Khlebtsov N, Dykman L. Chem Soc Rev, 2011, 40: 1647-1671

23 Yen HJ, Hsu SH, Tsai CL. Small, 2009, 5: 1553-1561

24 Sun YN, Wang CD, Zhang XM, Ren L, Tian XH. JNanosci Nanotech, 2011, 11: 1210-1216

25 Kah JCY, Grabinski C, Untener E, Garrett C, Chen J, Zhu D, Hussain SM, Hamad-Schifferli K. ACS Nano, 2014, 8: 4608-4620

26 Chen Y, Wang Z, Xu M, Wang X, Liu R, Liu Q, Zhang Z, Xia T, Zhao J, Jiang G, Xu Y, Liu S. ACS Nano, 2014, 8: 5813-5825

27 Guo W, Zhang S, Chen Y, Zhang D, Yuan L, Cong H, Liu S. Acta Biochim Biophys Sin, 2015, 47: 703-715

28 Guo W, Zhang S, Liu S. Oncol Rep, 2015, 33: 2992-2998

29 de Puig H, Tam JO, Yen CW, Gehrke L, Hamad-Schifferli K. J Phys Chem C, 2015, 119: 17408-17415

30 Ma J, Li R, Qu G, Liu H, Yan B, Xia T, Liu Y, Liu S. Nanoscale, 2016, 8: $18070-18086$

31 Ma J, Liu R, Wang X, Liu Q, Chen Y, Valle RP, Zuo YY, Xia T, Liu S. ACS Nano, 2015, 9: 10498-10515

32 Mosmann T. J Immunol Methods, 1983, 65: 55-63

33 Su YH, Ke YF, Cai SL, Yao QY. Light Sci Appl, 2012, 1: e14

34 Adair JH, Parette MP, Altınoğlu EI, Kester M. ACS Nano, 2010, 4: $4967-4970$
35 Chithrani BD, Ghazani AA, Chan WCW. Nano Lett, 2006, 6: 662-668

36 Vácha R, Martinez-Veracoechea FJ, Frenkel D. Nano Lett, 2011, 11: 5391-5395

37 Ferrari M. Nat Rev Cancer, 2005, 5: 161-171

38 Lacerda SHDP, Park JJ, Meuse C, Pristinski D, Becker ML, Karim A, Douglas JF. ACS Nano, 2010, 4: 365-379

39 Cigler P, Lytton-Jean AKR, Anderson DG, Finn MG, Park SY. Nat Mater, 2010, 9: 918-922

40 Tian M, Ogawa K, Wendt R, Mukhopadhyay U, Balatoni J, Fukumitsu N, Uthamanthil R, Borne A, Brammer D, Jackson J, Mawlawi O, Yang B, Alauddin MM, Gelovani JG. J Nucl Med, 2011, 52: 934-941

41 Kreyling WG, Abdelmonem AM, Ali Z, Alves F, Geiser M, Haberl N, Hartmann R, Hirn S, de Aberasturi DJ, Kantner K, Khadem-Saba G, Montenegro JM, Rejman J, Rojo T, de Larramendi IR, Ufartes R, Wenk A, Parak WJ. Nat Nanotech, 2015, 10: 619-623

42 Liang S, Li C, Zhang C, Chen Y, Xu L, Bao C, Wang X, Liu G, Zhang F, Cui D. Theranostics, 2015, 5: 970-984

43 Liu J, Zheng X, Gu Z, Chen C, Zhao Y. Nanomed-Nanotechnol Biol Med, 2016, 12: 486-487

44 Liu J, Zheng X, Yan L, Zhou L, Tian G, Yin W, Wang L, Liu Y, Hu Z, Gu Z, Chen C, Zhao Y. ACS Nano, 2015, 9: 696-707

45 Wang S, Shang L, Li L, Yu Y, Chi C, Wang K, Zhang J, Shi R, Shen H, Waterhouse GIN, Liu S, Tian J, Zhang T, Liu H. Adv Mater, 2016, 28: 8379-8387

46 Liu Y, Ai K, Liu J, Deng M, He Y, Lu L. Adv Mater, 2013, 25 : 1353-1359

47 Liu H, Liu T, Wu X, Li L, Tan L, Chen D, Tang F. Adv Mater, 2012, 24: $755-761$

48 Liu J, Wang P, Zhang X, Wang L, Wang D, Gu Z, Tang J, Guo M, Cao M, Zhou H, Liu Y, Chen C. ACS Nano, 2016, 10: 4587-4598

49 Ren W, Liu JY, Guo SJ, Wang EK. Sci China Chem, 2011, 54: 1334-1341

50 Tong LM, Zhu T, Liu ZF. Sci China Ser B, 2007, 50: 520-525

51 Indrasekara ASDS, Meyers S, Shubeita S, Feldman LC, Gustafsson T, Fabris L. Nanoscale, 2014, 6: 8891-8899

52 Tian F, Bonnier F, Casey A, Shanahan AE, Byrne HJ. Anal Methods, 2014, 6: 9116-9123

53 Song C, Yang B, Yang Y, Wang L. Sci China Chem, 2016, 59: 16-29

54 Michota A, Bukowska J. J Raman Spectrosc, 2003, 34: 21-25 